\title{
A Variation Level Set Formulation Local Based C-V Model for Medical Images Segmentation
}

\author{
Yan Yu, Chaobing Huang \\ Key Laboratory of Fiber Optic Sensing Technology and \\ Information Processing, Ministry of Education \\ Key Laboratory of Broadband Wireless Communications \\ and Sensor Networks, Hubei Province \\ School of Information Engineering, Wuhan University of \\ Technology, Wuhan, Hubei, 430070, P.R. China \\ E-mail:yuyanqs2008@163.com, huangcb@whut.edu.com
}

\author{
Ling Li \\ Key Laboratory of Robotics and Intelligent System of \\ Guangdong Province \\ Shenzhen Institutes of Advanced Technology, Chinese \\ Academy of Science (SIAT) \\ Shenzhen, Guangdong, 518055, P. R. China \\ E-mail: ling.li@siat.ac.cn
}

\begin{abstract}
Local image information is crucial for accurate segmentation of images with intensity inhomogeneity which usually occurs in medical images. However, image information in local region is not incorporated in popular region-based active contour models, such as piecewise constant models and piecewise smooth models. In this paper, a method which is able to use local information is proposed. The main point is the introduction of the local fitting information expressed by a kernel function which is crucial for segmentation. Our method is based on piecewise constant Chan-Vese model, and compare with different methods for several synthetic images and medical images.
\end{abstract}

Keywords- GACM, mediccal image segmentation, intensity imhomogeneity, $C-V$ model.

\section{INTRODUCTION}

Since active contour models (ACM) firstly introduced by kass et al[1], which are based on the theory of surface evolution and geometric flows, have been widely studied and successfully used in image segmentation field. Usually, ACM can be divided into two classes: parametric active contour models (PACM)[1] and geometric active contour models (GACM), GACM is proposed by Caselles et al[2]. PACM have been studied for many years which will not study in this paper; While GACM based on curve evolution theory and level set method have been a hot topic in recently years due to capability of processing images with intensity inhomogeneity which often occurs in medical images.

The main idea of GACM are: expressing contours as the zero level set of an implicit function defined in a higher dimension, usually refer as the level set function, and to evolve the level set function according to a partial differential equation (PDE)[3]. This approach shows several advantages over the conventional PACM. Firstly, the contours represented by the level set function may break or merge naturally during the evolution, and the topological changes are thus automatically handled. Secondly the level set function always remains a function on a fixed grid, which allows efficient numerical schemes[3] to solve

The existing GACM can be categorized into two major classes: edge-based and region-based. Edge-based uses edge information to attract the active contour toward the object boundary. Region-based aims at identifying each region of interest by using a certain region-based descriptor to guide the motion of the active contours. However, mostly popular region-based models tend to rely on intensity homogeneity in each region.

As referred above, intensity inhomogeneity often occurs in MR, CT, X-ray and US images, In references, intensity inhomogeneity can be addressed by more complex models than PC model[3]. For example, PS model, LBF[4] model, LIF model[5]. Following is this paper in detail.

\section{BACKGROUND}

\section{A. Mumford-Shah Functional for Image Segmentation}

In reference[6], Mumford and Shah formulated the optimal piecewise smooth approximation function $\mathrm{u}$ of image I, which varies smoothly within each sub-region of image domain, and rapidly or discontinuously goes around the boundaries, they proposed the following energy functional[6].

$E^{M S}(u, C)=\int_{\Omega}(u-I)^{2} d x+\mu \int_{\Omega / C}|\nabla u|^{2} d x+v|C|, x \in \Omega$

Where $|C|$ is the length of the contour $\mathrm{C}, \mu, v>0$ are fixed parameters. The unknown set $\mathrm{C}$ and the non-convexity of the above energy function make it difficult to be minimized. Several methods have been proposed to modify above function, including popular ones introduced below.

\section{B. The Piecewise Constant C-V Model}

In reference[3] Chan and Vese proposed an ACM based on the Munford-Shah model. Let $I$ be an output image and $\mathrm{C}$ be a closed curve, the energy functional is defined by:

$$
\begin{gathered}
E^{C V}\left(C, c_{1}, c_{2}\right)=\mu \cdot \text { length }(C)+ \\
\lambda_{1} \int_{\text {inside }(C)}\left|I-c_{1}\right|^{2} d x+\lambda_{2} \int_{\text {outside }(C)}\left|I-c_{2}\right|^{2} d x
\end{gathered}
$$

Where $\mu \geq 0, v \geq 0, \lambda_{1}, \lambda_{2}>0$ are fixed parameters. The Euclidean length term is used to regularize the contour: $C_{1}$ and $c_{2}$ are two constants that approximate the image intensities inside and outside the contour $C$, respectively. Minimizing the above energy functional by using steepest descent method, representing contour $C$ with zero level 
set $C=\{x \in \Omega \mid \phi(x)=0\}$ following is variation function:

$$
\begin{aligned}
& c_{1}(\phi)=\frac{\int_{\Omega} I H(\phi) d \Omega}{\int_{\Omega} H(\phi) d \Omega}, c_{2}(\phi)=\frac{\int_{\Omega} I(1-H(\phi)) d \Omega}{\int_{\Omega}(1-H(\phi)) d \Omega} \\
& \frac{\partial \phi}{\partial t}=\delta(\phi)\left[\mu \operatorname{div}\left(\frac{\nabla \phi}{|\nabla \phi|}\right)-\lambda_{1}\left(I-c_{1}\right)^{2}+\lambda_{2}\left(I-c_{2}\right)^{2}\right]
\end{aligned}
$$

The date fitting term $-\lambda_{1}\left(I-c_{1}\right)^{2}+\lambda_{2}\left(I-C_{2}\right)^{2}$ plays a key role in curve evolution, $\mu$ governs the tradeoff between the first and the second term. In most cases, we set $\lambda_{1}=\lambda_{2}$ and $\mu$ is a scaling parameter, if small enough, then small objects are likely to be extracted; if it is large, big objects can be detected.

Obviously, $C_{1}$ and $C_{2}$ are related to the global properties of the image. However, such global image information is not accurate if the image intensity inside or outside the contour is inhomogeneous. Figure 1. shows the above model results compared with several conventional method. We can see that the C-V model obtain better results than the threshold method, but not the promising results.

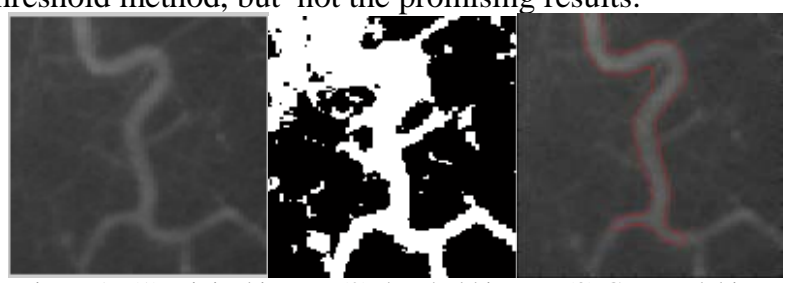

Figure 1.. (1) original image; (2) threshold image; (3) C-V model image. We can see that this level set method based on MumfordShah model can choose the initial contour everywhere in whole image, and not rely on the gradient, especially for images with noise it can achieve more effective results.

\section{OUR PROPOSED LOCAL C-V MOdEL BASED ON LOCAL DOMAIN INFORMATION.}

\section{A. The Local Domain C-V Model}

The C-V model just uses the global information so as not to segment image with inhomogeneity intensity problems. Here we detail the characteristics of the inhomogeneous intensity images, and give out the crucial information of this class images[3]: local information, incorporate this information into the $\mathrm{C}-\mathrm{V}$ model to realize accurate segmentation, named local based $\mathrm{C}-\mathrm{V}$ model.

Given a image $I$, its domain of definition is $\Omega$, Set $\mathrm{C}$ is a contour in the domain of $\Omega, x$ is an arbitrarily point, the energy function is below.

$$
\begin{gathered}
E^{C V}\left(C, c_{1}, C_{2}\right)=\lambda_{1} \int_{\text {inside }(C)}\left|I-C_{1}(x)\right|^{2} d x \\
+\lambda_{2} \int_{\text {outside }(C)}\left|I-C_{2}(x)\right|^{2} d x+\mu L(\mathrm{C})
\end{gathered}
$$

both of $\lambda_{1}, \lambda_{2}$ can adjust to obtain good results. Among these, notice that $C_{1}(x)$ and $C_{2}(x)$ are different from $C_{1}$ and $C_{2} . C_{1}$ and $C_{2}$ in $\mathrm{C}-\mathrm{V}$ model is the mean intensity of the images inside and outside of the contour $C$, respectively. However in our proposed method, $c_{1}(x)$ and $c_{2}(x)$ are two functions not constants whose values are decided by local information. $c_{1}(x)$ is the mean intensity of the points which is inside the circle of $x$ as the origin and $r$ as the radius and in the domain of contour $C$; relatively, $c_{2}(x)$ is the mean intensity of the points which is outside the circle of $X$ as the origin and $r$ as the radius and in the domain of contour $C$, namely:

$$
\begin{aligned}
& c_{1}(x)=\frac{\left[I(x) H_{v}(\phi(x))\right]^{*} K r(x)}{H_{v}(\phi(x))^{*} K r(x)} \\
& c_{2}(x)=\frac{\left[I(x)\left(1-H_{v}(\phi(x))\right)\right]^{*} K r(x)}{\left(1-H_{v}(\phi(x))\right) * K r(x)}
\end{aligned}
$$

Among this, * is the Convolution symbols, $\operatorname{Kr}(x)$ is Kernel function proposed in this paper.

$$
K r(x)=\frac{1}{2}\left(\operatorname{sign}\left(r^{2}-x^{2}\right)+1\right)
$$

Where $r$ is the radius of the Kernel function. For the kernel function when the absolute value of $x$ is smaller than radius of $r$, the value is 1 , when the absolute value of $x$ is larger than radius of $r$, the value is 0 . The meaning of introduced Kernel function is: $\operatorname{Kr}(x)$ making the mean intensity of $x$ is just relative to the intensity of the points nearby $x, c_{1}(x)$ and $c_{2}(x)$ making the constant corresponding to every points instead by a local function corresponding to every points.

In this method, the local energy of point $\mathrm{x}$ is decided by the local information of point $x$, on the basis of which will overcome $\mathrm{C}-\mathrm{V}$ model using the global information with the disadvantages of segmenting the intensity inhomogeneity images. Obviously, in equation(4), when the contour C locate at the boundary of objects, the energy function $E$ is smallest. As a result, we can minimize the energy function in the full domain of images to get the exact position of contour $\mathrm{C}$, namely the exact position of the edges of objects.

To process the energy function into the level set function so that it can automatically process arbitrary topology changes of contour curves (such as curve division and merger). In order to construct variation level set model, we introduce the Heaviside function $H(\phi)$ and Dirac function $\sigma(\phi)$, change the equation into level set function.

$$
\begin{aligned}
& E\left(\phi, c_{1}, C_{2}\right)=\lambda_{1} \int_{\Omega}\left|I(x)-C_{1}(x)\right|^{2} H(\phi) d x+ \\
& \lambda_{2} \int_{\Omega}\left|I(x)-c_{2}(x)\right|^{2}(1-H(\phi)) d x+\mu L(\phi)
\end{aligned}
$$


Where the length of zero level set contour is:

$$
L(\phi)=\int_{\Omega} \sigma(\phi(x)|\nabla \phi(x)| d x
$$

In order to ensure stable evolution of the level set function, we add the distance regularizing term in $\mathrm{Li}$ et al.'s variation level set formulation[7] to penalize the deviation of the level set function from a signed distance function. The deviation of the level set function from a signed distance function is characterized by the following intergral:

$$
E_{\text {cor }}(\phi)=\int_{\Omega} \frac{1}{2}(\mid \nabla \phi(x)-1)^{2} d x
$$

In practice, the Heaviside function and Dirac function are approximated by smooth functions $H_{v}(x)$ and $\sigma_{v}(x)$.

$$
\begin{aligned}
& H_{v}(x)=\frac{1}{2}\left[1+\frac{2}{\pi} \arctan \left(\frac{x}{v}\right)\right] \\
& \sigma_{v}(x)=\frac{v}{\pi\left(v^{2}+x^{2}\right)}
\end{aligned}
$$

So that, we obtain the whole energy function:

$$
F\left(\phi, c_{1}, c_{2}\right)=E\left(\phi, c_{1}, c_{2}\right)+\gamma E_{\text {cor }}(\phi)
$$

This is the energy functional we will minimize to find the object boundary.

$$
\begin{aligned}
& F\left(\phi, c_{1}, c_{2}\right)=\lambda_{1} \int_{\Omega}\left|I(x)-c_{1}(x)\right|^{2} H(\phi) d x+\lambda_{2} \int_{\Omega}\left|I(x)-c_{2}(x)\right|^{2} \\
& (1-H(\phi)) d x+\mu \int_{\Omega} \sigma\left(\phi(x)|\nabla \phi(x)| d x+\gamma \int_{\Omega} \frac{1}{2}(\mid \nabla \phi(x)-1)^{2} d x\right.
\end{aligned}
$$

\section{B. Gradient Descent Flow for Calculate Level Set Energy Function}

We use standard gradient descent (or steepest descent) method[4] to minimize the energy function. The derivation of the gradient flow is similar to that of the PC and PS models in [7]. Following is detailed procedure.

For the fixed level set function, we minimize the function in (12) with respect to $H_{v}$ and $\phi$. By calculus of variations, it can be shown that the function are given by the equations of (5). Note that the denominators in (5) always positive, due to the fact that $1-H_{v}(\phi)>0$ and $H_{v}(\phi)>0$ by the definition of equation (10).

Keeping $c_{1}(x), c_{2}(x)$ fixed, minimizing energy function (12) with respect to $\phi$,derive the gradient descent flow [9]:

$$
\begin{gathered}
\frac{\partial \phi}{\partial t}=-\sigma_{v}(\phi)\left[\lambda_{1}\left(I-c_{1}\right)^{2}-\lambda_{2}\left(I-c_{2}\right)^{2}\right]+ \\
\mu \sigma_{v}(\phi) \operatorname{div} \frac{\nabla \phi}{|\nabla \phi|}+\gamma\left(\Delta \phi-\operatorname{div} \frac{\nabla \phi}{|\nabla \phi|}\right)
\end{gathered}
$$

Where the Laplace operator is : $\Delta \phi=\nabla(\nabla \phi)$

In above model we introduce the keys of intensity inhomogeneity in images: local domain information. The energy function based on this prior knowledge is able to accurately express the characteristics of the different tissues images with intensity inhomogeneity to ensure results. In process of evoluing level set function incorporating energy distortion function(12), ensure the level set function keeping as the signed distance function to avoid initialization.

\section{IMPLEMENTATION RESULTS AND ANALYSIS.}

The environment is Matlab2010b, Sony, CPU 2.93 Ghz, RAM 2G. Our proposed model is implemented on synthetic images, X-ray vessel images, MR images and compare with PC model in reference[3] and LBP model in reference[4] to verify the effectiveness.

\section{A. Implementation with Several Medical Images with severe intensity imhomogeneity}

Figure. 2 shows the results on vessel images and brain images, the initial level set is computed from the region enclosed by the quadrilateral enclosing the same as in reference[5]. For the two group images, we set the parameters $\lambda_{1}=\lambda_{2}=1, \mu=0.01 * 255 * 255, \gamma=1.0 ; r=3$, and timestep is 0.1 , iteration 500 . From the comparison with different methods with intensity inhomogenous images, PC model lose some important parts, the LBP model and Our method almost the same results while our method is more accurate with more small parts in the interest region.
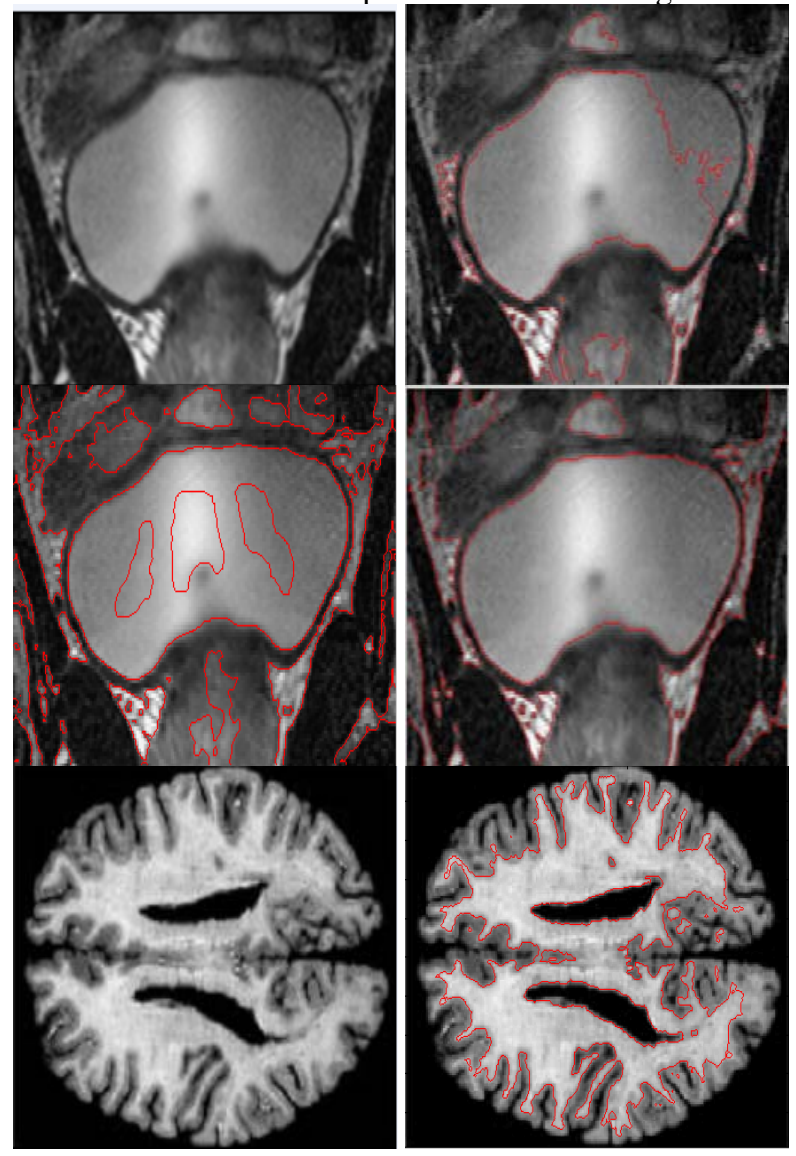

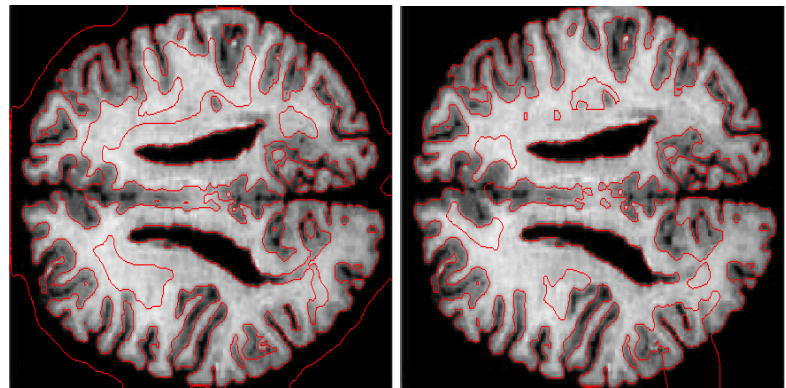

Figure 2. Above are X-ray, and MR images. In each group the first are original images, the second are $\mathrm{C}-\mathrm{V}$ model; the third are LBP model result; the fourth are our proposed method results.

\section{B. Implementation Images including Several Objects}

In Figure.3, there are MR cell and synthetic images. For the two group images, we set the parameters $\lambda_{1}=\lambda_{2}=1$, $\mu=0.01 * 255 * 255, \gamma=1.0 ; r=3$, and timestep is 0.1 , and iteration is 500. From the comparison results of different methods, we can see that when there are several objects our method will not get promising results due to it only use one level set.
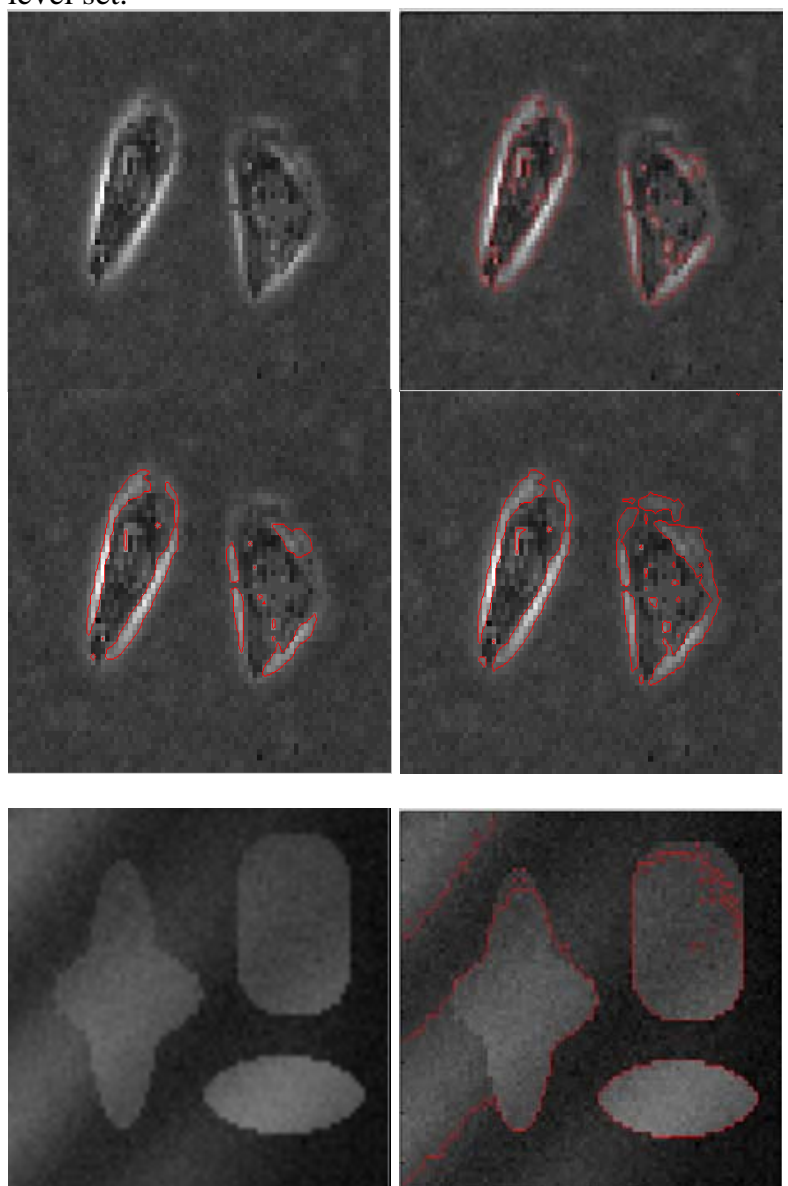

Figure 3. Above are MR and synthetic images.In each image,there are more than one object to be segmented. In each group, the first are original images, the second are $\mathrm{C}-\mathrm{V}$ model results, the third are LBP model results; the fourth are our proposed method results.
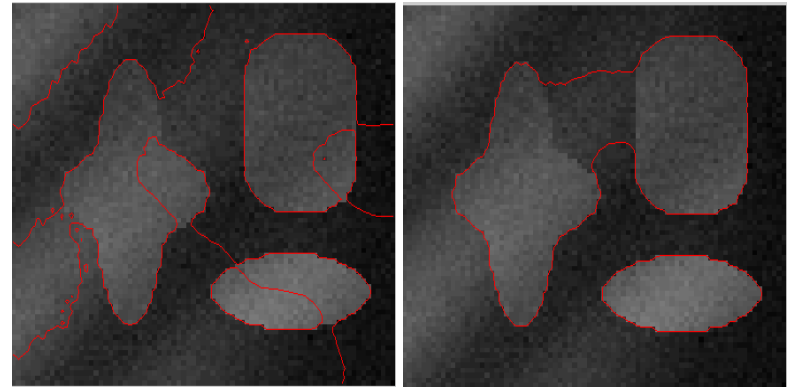

\section{CONCLUSIONS AND FUTURE WORKS}

In this paper, a new variation level set formulation is presented that incorporates local intensity information and without re-initialization. The proposed method can obtain more accurate segmentation results. However, it still has following problems: (1) When there are several objects our method is better than the method proposed in references, but it still don't get promising results; (2) For some images with noise we should introduce filtering method as the preprocessing step[9]. Given that we need further research, and in the future we can extend the method to threedimension images.

\section{ACKOWNLEDGMENTS}

This paper is supported by the following grants: Teaching and Research Project of Hubei Province (2010092), National Natural Science Foundation of China (61103165), Shenzhen Key Laboratory Project (CXB201005260056A), and ShenZhen Distinguished Young Scholars Fund (JC201005260248).

\section{REFERENCES}

[1] Kass M, Witkin A, and Terzopoulos D," Snakes: active contour models,” Int J Comput Vis 1987;1(4):321-31.

[2] V.Caselles,F.Catte,T.Coll, and F.Dibos, "A geometric model for active contours in image processing", Number. Math., Vol.66, pp.131,1993.

[3] Tony F.Chan, and Luminita A.Vese, "Active Contours without Edges," Proc. IEEE Transaction on Image Processing (TIP 01), Vol.10.NO.2, February 2001.

[4] Chunming Li, Chiu-Yen Kao, John C.Gore, and Zhaohua Ding, "Implicit Active Contours Driven by Local Binary Fitting," Proc. IEEE Computer Society Conference on Computer Vision and Pattern Recognition(CVPR 07), 2007.

[5] Kaihua Zhang, Huihui Song, Lei Zhang,"Active contours driven by local image fitting energy,” Pattern Recognition 43(2010)1199-1206.

[6] Mumford D, Shah J, "Optimal approximations by piecewise smooth functions and associated variational problems," Commum Pure Appl Math, 1989, 42:577-685.

[7] Chunming Li, Chenyang Xu, Changfeng Gui, Martin D.Fox, ”Level Set Evolution without Re-initialization: A new Variational Formulation," Proc. IEEE Computer Society Conference on Computer Vision and Pattern Recognition(CVPR 05), 2005.

[8] Tony F.Chan, and Luminita A.Vese," Active Contours without Edges," Proc. IEEE Transaction on Image Processing (TIP 01), Vol.10. NO.2, February 2001.

[9] Fu Shu-jun, Ruan Qiu-qi, Li Yu, and Wang Wen-qia, “Anisotropic Diffusion Equation Based Ultrasonic Image Denoising and Edge Enhancement,” Acta Electronic Sinica. Vol.33 No.7, July 2005. 